\title{
Ultrasonographic observation from pre-catheterization to the development of anticancer drug-induced induration: A case report
}

\author{
Mari Abe-Doi ${ }^{1}$, Ryoko Murayama ${ }^{1}$, Chieko Komiyama ${ }^{2}$, and Hiromi Sanada ${ }^{3}$ \\ ${ }^{1}$ Department of Advanced Nursing Technology, graduate school of medicine, the University \\ of Tokyo \\ ${ }^{2}$ The University of Tokyo Hospital \\ ${ }^{3}$ The University of Tokyo Graduate School of Medicine Division of Health Science and \\ Nursing Department of Gerontological Nursing Wound Care Management
}

June 22, 2020

\begin{abstract}
This case showed that anticancer drug administration induces unhealthy subcutaneous tissue (thrombus or edema) without subjective symptoms, abnormal sign by palpation or inspection, which have an extravasation risk. Especially, short-term interval therapy such as a weekly chemotherapy have high risk for anticancer drug infusion to the risky site.
\end{abstract}

Ultrasonographic observation from pre-catheterizationto the development of anticancer druginduced induration: A case report

\section{Authors}

Mari Abe-Doi, MHS, RN 1, Ryoko Murayama, PhD, RN, RNM 1,2, Chieko Komiyama, MNS,RN, 3, and Hiromi Sanada, PhD, RN, WOCN, FAAN, 2,4

1 Department of Advanced Nursing Technology, Graduate School of Medicine, the University of Tokyo, Bunkyo-ku, Tokyo

2 Global Nursing Research Center, Graduate School of Medicine, the University of Tokyo, Bunkyo-ku, Tokyo

3 Department of Nursing, The University of Tokyo Hospital, Bunkyo-ku, Tokyo

4 Department of Gerontological Nursing/Wound Care Management, Graduate School of Medicine, the University of Tokyo, Bunkyo-ku, Tokyo

Corresponding author

Hiromi Sanada, PhD, RN, WOCN, Department of Gerontological Nursing/Wound Care Management, Graduate School of Medicine, the University of Tokyo, 7-3-1 Hongo, Bunkyo-ku, Tokyo, 113-0033, Japan. Email: hsanada-tky@umin.ac.jp, 81-3-5841-3419

\section{Key clinical message}

This case showed that anticancer drug administration induces unhealthy subcutaneous tissue (thrombus or edema) without subjective symptoms, abnormal sign by palpation or inspection, which have an extravasation risk. Especially, short-term interval therapy such as a weekly chemotherapy have high risk for anticancer drug infusion to the risky site.

\section{Key words}


oncology nursing; peripheral venous; induration; chemotherapy; injection site reaction

\section{Introduction}

Some practice guidelines do not recommend the use of a peripheral intravenous catheter for irritant or vesicant continuous administration to avoid vessel damage [1,2]. However, peripheral intravenous catheters are unavoidably used in some patients for drug administration because of their conditions, such as superior vena cava syndrome, coagulopathy, or compromised condition. In clinical settings, induration can be observed at the catheterization sites after anticancer drug administration even without obvious signs and symptoms of extravasation such as feelings of tingling, burning, pain, swelling, and redness at the injection site [2]. If induration is found by physicians or nurses, inserting catheter to the affected site is avoided. It can lead to catheter placement in inappropriate sites, such as the hands, near joints, or thin fragile veins [3]. These catheterization sites are reported as risk factors of extravasation. Thus, induration after anticancer drug administration is a clinical challenge for patients who must receive repetitive treatment using a peripheral intravenous catheter; induration occurrence should be prevented. It is important to know the actual condition of induration very well for safe chemotherapy treatment. Ultrasonography is used to observe the condition of the vein or surrounding tissue [4,5]. We already reported actual condition of induration through ultrasonography after obvious extravasation [6], but the onset process of induration without obvious extravasation was unclear. Here, we report time-course observation of the catheterization site from pre-catheterization to induration development through ultrasonography (Figure 1).

\section{Case Description}

A 77-year-old man with esophagus cancer was receiving chemotherapy, paclitaxel, weekly through a peripheral intravenous catheter at a chemotherapy room in a university hospital in Japan. Data of patient character and details of drugs administration method were collected from his medical chart.

No abnormal subcutaneous tissue condition was observed at the target site, which was confirmed through ultrasonography, prior to catheterization for the administration anticancer drug; on the second week of paclitaxel administration, diameter of the target vein was $2.4 \mathrm{~mm}$. It was calculated by measuring the minor axis and major axis three times, and measurements were averaged. Then the average of the minor axis was added to the average of major axis and divided by two.

All ultrasound images were obtained by one researcher using a Noblus@ $\mathrm{R}$ with linear array (5-18 MHz) 2D probe (Hitachi, Ltd, Medical, Tokyo, Japan). Pre-catheterization images were obtained by a 5-cm swept probe (peripheral side $2.5 \mathrm{~cm}$; center side $2.5 \mathrm{~cm}$ ) with short axis over the point that nurses selected as catheterization site, along the vein. Ultrasonographic images were assessed with reference to previous studies $[4,5]$; although unclear, superficial fascia at surrounding vein was judged as subcutaneous edema presence, and ununiform echoic area in the intravascular lumen was judged as thrombus.

The nurse inserted a $24 \mathrm{G}$ catheter at the patient's cephalic vein on the right forearm. Location of the catheterization site was recorded by a measure and digital camera. Drug administration protocol was as follows: First, the nurse secured the catheterization site by administering normal saline solution; a $6.6 \mathrm{mg}$ of dexamethasone sodium phosphate $+20 \mathrm{mg}$ of famotidine $+5 \mathrm{mg}$ of d-Chlorpheniramine Maleate $+50 \mathrm{~mL}$ of normal saline were administered at $300 \mathrm{~mL} / \mathrm{hr}$. for $10 \mathrm{~min}$. Next, $50 \mathrm{~mL}$ normal saline was administered at $300 \mathrm{~mL} / \mathrm{hr}$. for $10 \mathrm{~min}$. Then, $156 \mathrm{mg}$ paclitaxel $+50 \mathrm{~mL}$ normal saline were administrated at $300 \mathrm{~mL} / \mathrm{hr}$. for $60 \mathrm{~min}$. Lastly, normal saline $30 \mathrm{~mL}$ at $300 \mathrm{~mL} / \mathrm{hr}$. for $6 \mathrm{~min}$ was administered. Thus, total drug administration time was about $90 \mathrm{~min}$. Anticancer drug administration was completed without local adverse event or discomfort such as swelling, redness, and pain; however, subcutaneous edema and thrombus were observed through ultrasonography just after finishing anticancer drug administration (Figure 2; A, B).

One week later, no abnormal findings at the catheterization site were noted upon inspection or palpation. However, subcutaneous edema and the remaining thrombus at the site were observed, which were confirmed through ultrasonography. Three weeks later, the site was positively assessed for induration by palpation, and subcutaneous tissue and thrombus were observed through ultrasound. Four weeks later, the induration, 
thrombus, and subcutaneous edema were still present (Figure 2; C, D, E). No symptoms were observed upon inspection at any one time, and no subjective symptoms such as pain or sensations of burning were reported.

On the post treatment days, ultrasonographic images were taken same way as pre-catheterization, using puncture site or induration site as a center point. Evaluation of the presence of induration through palpation was conducted by a clinical nurse and one researcher who had clinical experience as a nurse.

This observation was conducted in accordance with the Declaration of Helsinki, and study protocol was approved by the Research Ethics Committee of the facility where researchers belong (No. 11650-1). Using images and information for report was permitted by the patient. Observation period was from February to March in 2019.

\section{Discussion and conclusion}

We observed the target site from pre-catheterization time until the development of induration caused by chemotherapy drug administration through a peripheral intravenous catheter. This is the first study that reported time-course observation of the catheterization site from pre-catheterization to induration development through ultrasonography.

During pre-catheterization, no abnormal findings were observed in the subcutaneous tissue and vein, and its diameter was enough to insert a $24 \mathrm{G}$ catheter [7]. There was no complication during drug administration, but subcutaneous edema and thrombus were confirmed upon finishing administration. A week later, induration was not observed at the site, even with the presence of subcutaneous edema and thrombus. However, induration with edema or thrombus was observed 3 weeks later. Above these reasons, induration caused by anticancer drug administration was due to the fibrotic subcutaneous tissue formation, which was a result of mild inflammation that did not induce pain, swelling, and erythema.

Fibrosis which is the last part of inflammation reaction is formed gradually and lasts about a few weeks [8]. Thus, we consider that fibrosis had still not become advanced at one-week post chemotherapy. It means that in short treatment interval chemotherapy, there is possibility of anticancer drug administration using the site that has subcutaneous edema or thrombus as catheter placement site because nurses cannot detect abnormal tissue condition by palpation only.

"Unhealthy subcutaneous tissue" is one of the extravasation risk factors [3]. This case showed that anticancer drug administration through a peripheral intravenous catheter induces the development of unhealthy subcutaneous tissue (thrombus or edema) without subjective symptoms, abnormal sign by palpation or inspection. Also, this case showed how subsequent anticancer drugs, including vesicants, may be infused at vulnerable sites. Continuous administration of irritants or vesicants via a central vein is recommended. However, for patients who unavoidably require peripheral intravenous catheters, the catheterization site must be carefully selected. Short-term interval therapy such as a weekly chemotherapy have especially high risk for anticancer drug infusion at risky sites. Future researchers should consider the possibility of these risky sites when selecting appropriate catheterization site.

\section{Author Contributions :}

MA: designed the research; MA and CK: contributed data collection; MA: drafted the manuscript; RM and HS: critically reviewed the manuscript and supervised the whole study process. All authors read and approved the final manuscript.

\section{Reference}

Gorski L., Hadaway L., Hagle M., McGoldrick M., Orr M., and Doellman D. Infusion Therapy Standards of Practice. 2016. J Infus Nurs. $39 \mathrm{Suppl} \mathrm{Jan./Feb.} \mathrm{1S}$

1. J. A. Pérez Fidalgo, L. García Fabregat, A. Cervantes, Margulies A., Vidall C., Roila F., on behalf of the ESMO Guidelines Working Group.2012. ESMO Guidelines Working Group. Management of 
chemotherapy extravasation: ESMO-EONS Clinical Practice Guidelines. Ann Oncol . 23 Suppl 7: vii167-73.

2. Onesti MG., Carella S., Fioramonti P., and Scuderi N. 2017. Chemotherapy Extravasation Management: 21-Year Experience. Ann Plast Surg. 79(5):450-457.

3. Yabunaka K., Murayama R., Takahashi T., Tanabe H., Kawamoto A., Oe M., et al. 2015. Ultrasonographic appearance of infusion via the peripheral intravenous catheters. Journal of Nursing Science and Engineering. 2(1):40-46.

4. Yabunaka K., Murayama R., Tanabe H., Takahashi T., Oe M., Oya M., et al. 2016. Ultrasonographic classification of subcutaneous edema caused by infusion via peripheral intravenous catheters. $J$ Med Ultrasound. 24(2):60-5.

5. Abe-Doi M., Murayama R., Yabunaka K., Tanabe H., Komiyama C., and Sanada H. 2019. Ultrasonographic assessment of an induration caused by extravasation of a nonvesicant anticancer drug: A case report.Medicine. 98(14): e15043.

6. Tanabe H., Takahashi T., Murayama R., Yabunaka K., Oe M., Matsui Y., et al. 2016. Using Ultrasonography for Vessel Diameter Assessment to Prevent Infiltration. J Infus Nurs. 39(2):105-11.

7. Profyris C., Tziotzios C., and Do Vale I. 2012. Cutaneous scarring: Pathophysiology, molecular mechanisms, and scar reduction therapeutics Part I. The molecular basis of scar formation. J Am Acad Dermatol. 66(1):1-10

Figure legends

Figure 1: none

Figure 2:

A: Just before insertion a catheterization; Vein lumen area was imaged as anechoic area.

B: Just after completing anticancer administration, and just before the catheter removal; Two high echo points shown by arrow are upper wall and lower wall of the catheter. Some high echo points at skin surface are reflection by a catheter securement film. (Refer B': Illustration diagram) There was no anechoic area, instead, vein lumen was imaged as non-uniform echoic area. It was considered thrombus presence. Also, uncleared superficial fascia was observed at the surrounding tissue of the vein. It was considered subcutaneous edema.

C: Post treatment 1 week; Un-uniform echoic area remained in the vein lumen, and subcutaneous edema at surround tissue of the vein. There was nothing abnormal detected by palpation.

D: Post treatment 3 weeks; Un-uniform echoic area remained in the vein lumen, and subcutaneous edema at surround tissue of the vein. Furthermore, induration was found by palpation in the site.

E: Post treatment 4 weeks; Unechoed area become clear in the vein lumen, but there was still remain thrombus and subcutaneous edema. Also, induration remained. 


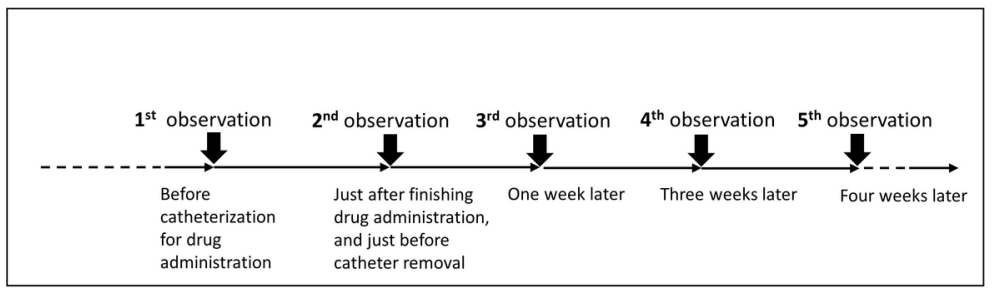

Figure 1. Time course of observation using an ultrasonography
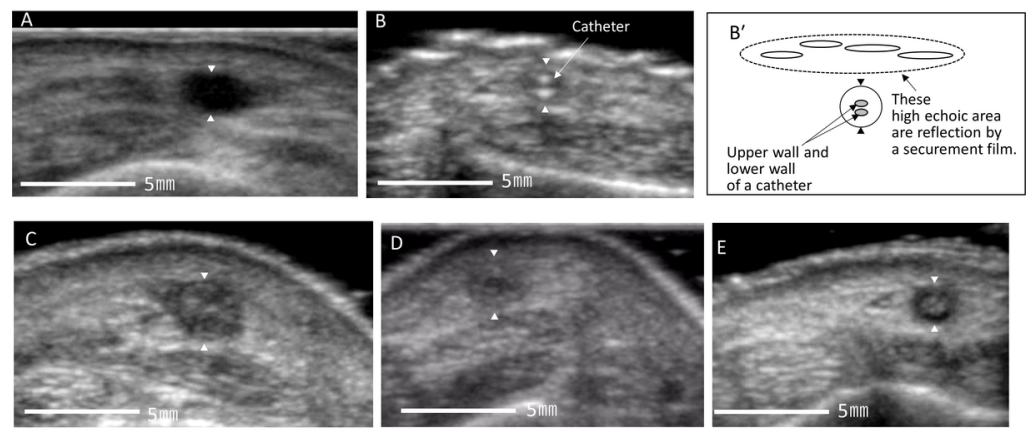

Figure 2. Catheterization site for anticancer administration : Arrowheads show the target vein 